\title{
Ultrasound contrast agent microbubbles with ultrahigh loading capacity of camptothecin and floxuridine for enhancing tumor accumulation and combined chemotherapeutic efficacy
}

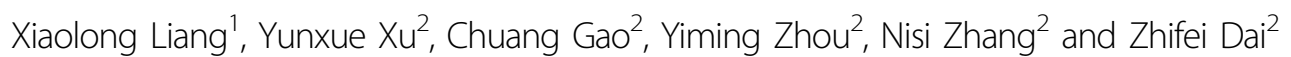

\begin{abstract}
In addition to the well-known use of microbubbles (MBs) as powerful contrast agents for general tissue delineation and perfusion in ultrasound (US) imaging, US-targeted MB destruction (UTMD) has been demonstrated to be an emerging technique for noninvasive drug delivery to tumor sites. However, the very limited drug-loading capacity of conventional MBs remains a great obstacle to their application as an efficacious cancer therapy. In this study, an amphiphilic Janus camptothecin-floxuridine (CF) conjugate was synthesized to engineer CF MBs with ultrahigh drugloading contents (up to $56.7 \pm 2.3 \%$ ). CF MBs were proven to be an excellent contrast agent to enhance US imaging for identifying the location and size of tumors. Upon local US exposure to burst CF MBs under the guidance of contrast-enhanced US imaging, successful conversion of CF MBs into CF NPs in situ resulted in 14 times higher drug accumulation of the CF conjugate via the sonoporation effect than that of CF NPs and CF MBs without US. After CF was internalized by tumor cells and its ester bond was hydrolyzed, camptothecin (CPT) and floxuridine (FUDR) were released at an exact 1:1 ratio to achieve coordinated pharmacokinetics, leading to significantly higher tumor growth inhibition in murine tumor models of CF MBs combined with US ( 72.4\%) than CF NPs ( 54.1\%) and liposomes loaded with CPT and FUDR ( 21.6\%). Overall, these results demonstrate that the combination of CF MBs with US is a powerful strategy for remarkably enhancing the combined chemotherapeutic efficacy and greatly reducing the undesirable side effects.
\end{abstract}

\section{Introduction}

Nanotherapy has attracted extensive interest for cancer treatments, mostly because of the uniquely appealing features of nanomedicines, for example, improved circulation, passive accumulation in tumors via the enhanced permeability and retention (EPR) effect of nanoparticles, and reduced toxicity ${ }^{1-4}$. Upon systemic administration for

\footnotetext{
Correspondence: Zhifei Dai (zhifei.dai@pku.edu.cn)

'Department of Ultrasound, Peking University Third Hospital, 100191 Beijing, China

${ }^{2}$ Department of Biomedical Engineering, College of Engineering, Peking University, 100871 Beijing, China
}

solid tumor treatment, nanoparticulate chemotherapeutic agents can be retained by the poorly developed lymphatic system in the tumor microenvironment and further release their drug payload ${ }^{5,6}$. Indeed, several nanomedicines, such as Abraxane, Mariqbo, Doxil, and DaunoXome, have been approved for cancer treatment by the FDA since 1999, mainly due to their improved side effect profile $^{7,8}$. Despite the great success of nanomedicines, their full potential has not been realized since high interstitial pressures need to be overcome when nanoparticles penetrate solid tumors ${ }^{9,10}$ and only up to $5 \%$ of the injected dose may accumulate at the tumor ${ }^{11,12}$. 
Although encapsulating drugs in nanoparticles has been widely shown to improve their pharmacokinetics and biodistribution, no marketed nanomedicines have yet exhibited an overall survival benefit in direct comparison with the conventional parent drug ${ }^{5-8,10}$. Therefore, there is still a pressing need to develop new strategies for cancer treatment ${ }^{13-15}$.

In addition to the well-known use of microbubbles (MBs) as powerful contrast agents for general tissue delineation and perfusion in ultrasound (US) imaging, MBs are widely explored to carry drugs for remotely controlling intratumoral drug release and enhancing intracellular drug accumulation by bursting MBs using a US beam with high precision and penetration depth ${ }^{16,17}$. Drugs can be introduced into the MBs by various methods, including binding drugs to the $\mathrm{MB}$ shell and attaching site-specific ligands. This approach, called UStargeted MB destruction (UTMD), utilizes the inertial acoustic cavitation of the MBs to cause sonoporation, greatly facilitating intracellular drug uptake at the target $^{18-21}$. However, the significantly limited surface area and shell thickness of MBs will hamper their effectiveness as drug carriers due to their very low loading capacity $^{18,22,23}$. To overcome this shortcoming, MBs functionalized with drug-loaded liposomes or other nanoparticles have been fabricated; however, they have shown problems with their complexity and stability ${ }^{24}$. Furthermore, an even greater limitation to the use of MBs for drug delivery is their micron size. Although the tumor vasculature may be leaky enough for even the MBs to

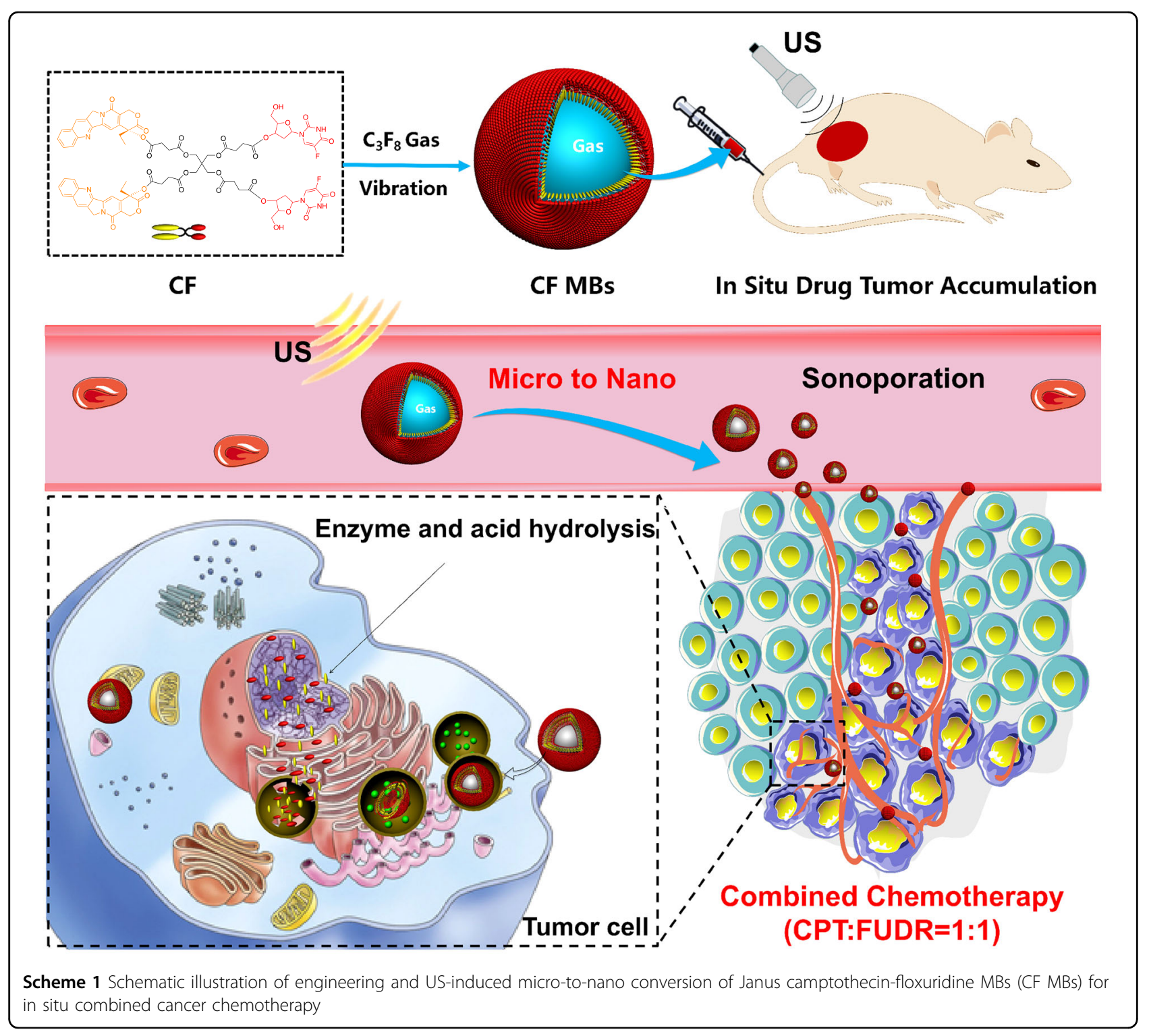


cross the endothelium, the distribution of sufficiently wide gaps is unpredictable, and therefore, a particle with smaller size would be preferable. In addition, nanoparticles can actively cause nanoparticle-induced endothelial leakiness (NanoEL), which depends on the nanoparticles and not on the tumor ${ }^{25-29}$. Therefore, a new drug delivery system combining the advantages of both MBs and nanoparticles is strongly needed to improve drug accumulation and penetration into the tumor site.

It has been reported that anticancer drug combinations simultaneously delivered to tumor cells can afford synergistic therapeutic effects, thus overcoming tumor heterogeneity and drug resistance issues. The combination of irinotecan and a fluoropyrimidine derivative in a synergistic 1:1 molar ratio inside a liposome has been demonstrated to provide greatly enhanced antitumor therapeutic efficacy ${ }^{30-}$ ${ }^{34}$. In this study, a highly symmetric Janus camptothecinfloxuridine $(\mathrm{CF})$ conjugate linked with two hydrophobic camptothecin (CPT) molecules and two hydrophilic floxuridine (FUDR) molecules and with multivalent pentaerythritol through hydrolyzable ester bonds was synthesized using a protection-deprotection strategy ${ }^{33-35}$. Its amphiphilic structure facilitates the self-assembly of CF MBs, enabling highly stable codelivery of drug combinations from conjugates with remarkably high drug-loading content (DLC), a fixed CPT/FUDR molar ratio of 1:1 and no premature release (Scheme 1).

When intravenously administered into tumor-bearing mice, CF MBs can circulate inside the bloodstream without extravasation to the normal tissues due to their micron size. More interestingly, CF MBs themselves can serve as contrast agents for enhanced US imaging not only to help visualize drug distribution and drug release at the target site but also to help identify the location and size of tumor. Under the guidance of US imaging, CF MBs circulating through the tumor site were locally disrupted at the tumor site upon exposure to lowintensity US and were in situ converted into CF NPs. Significantly increased amounts of CF NPs accumulated in the tumor site due to the enhanced permeation of the cell membrane and vasculature through the sonoporation effect. After CF NPs were internalized into tumor cells, the ester bond in the CF conjugate was hydrolyzed by esterases and the acid environment in tumor cells, and both CPT and FUDR were uniformly released, leading to significant improvement in the chemotherapeutic effect. CF MBs represent a strategy to deliver and preserve a fixed 1:1 molar ratio of the two drugs. This ratio shows synergistic anticancer activity both in vitro and in vivo. The integration of CF MBs and US is highly advantageous for the potential (pre-)clinical implementation of more efficient and less toxic treatment regimens for individual patients.

\section{Results and discussion}

\section{Design and engineering of CF MBs with ultrahigh drug- loading capacity}

Langmuir monolayers serve as a unique model for understanding the behavior and formation of MBs typically used as US contrast agents. The collapse pressure can be used to predict the stability of MBs under US and blood pressure. For the rational design of CF MBs, the behavior of CF monolayers was systematically studied using a Langmuir trough method by linking composition and processing history with phase behavior and microstructure. Figure 1a shows the surface pressure $(\pi)$ molecular area (A) isotherm of a CF film spreading on a water surface at $20^{\circ} \mathrm{C}$. The onset area of the spreading film is approximately $7 \mathrm{~nm}^{2}$ per CF molecule. Upon compression, the isotherm showed a plateau from approximately $25 \mathrm{~m} \mathrm{Nm}^{-1}$. A continuous increase in surface pressure was detected after the plateau region upon further compression. The molecular areas extrapolated from the linear part of the $\pi-A$ curves were approximately 1.5 and $6.5 \mathrm{~nm}^{2}$ molecule ${ }^{-1}$ before and after the plateau, respectively. By comparing the onset molecular area and the two extrapolated molecular areas, it can be concluded that the CF molecule is inclined with its equilateral plane on the water surface at the very beginning of the spreading. However, the molecule can easily be compressed and made to stand vertically on the water surface, with two hydrophilic FUDR molecules attaching to the water surface. When the film was compressed to the plateau region, a multilayer might be formed via collapse at $\sim 51 \mathrm{mN} \mathrm{m}^{-1}$. The reproducibility of the isotherm and high collapse pressure indicated that a stable film was formed when CF was spread on the subphase surface. To further investigate the interactions among the three MB-forming materials in a twodimensional monolayer, $\pi-\mathrm{A}$ isotherms for mixtures of CF/1,2-distearoyl-sn-glycero-3-phosphocholine (DSPC)/ 1,2-distearoyl-sn-glycero-3-phosphoethanolamine- $N$ [methoxy(polyethylene glycol)-2000] (DSPE-PEG2000) at different molar ratios were recorded in phosphatebuffered saline (PBS) (pH 7.4). As shown in Fig. 1a, all $\pi-\mathrm{A}$ isotherms showed a transition from an expanded phase to a condensed phase; for pure CF, the collapse pressure was found to be $\sim 51 \mathrm{mN} \mathrm{m}^{-1}$, while the binary phospholipid system $(0 \%$ CF, DSPC: DSPE-PEG2000 = 9: 1) collapsed at $\sim 63 \mathrm{mN} \mathrm{m}^{-1}$. All the $\pi-A$ isotherms of the mixed monolayers of CF/DSPC/DSPE-PEG2000 showed an expansion and retraction between those of the above two monolayers, and the collapse pressure showed a nonmonotonic change as the composition was varied. Compared with that of the binary phospholipid system, the collapse pressure first increased at lower CF content ( $\sim 58 \mathrm{mN} \mathrm{m}^{-1}$ for $10 \mathrm{~mol} \%$; $~ 61 \mathrm{mN} \mathrm{m}^{-1}$ for $\left.20 \mathrm{~mol} \%\right)$ as the CF molar ratio in the mixed monolayers increased and 

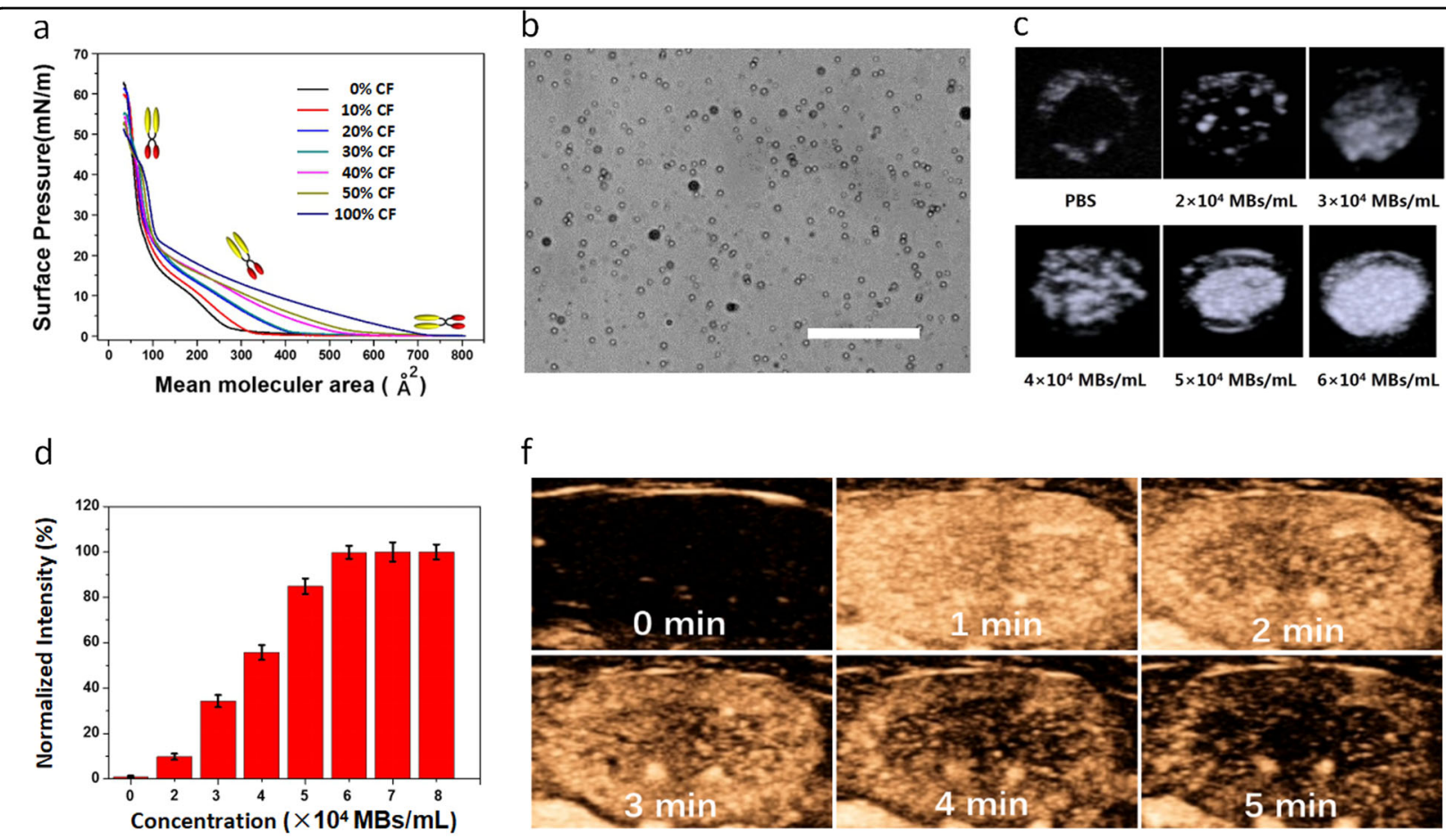

$4 \times 10^{4} \mathrm{MBs} / \mathrm{mL} \quad 5 \times 10^{4} \mathrm{MBs} / \mathrm{mL} \quad 6 \times 10^{4} \mathrm{MBs} / \mathrm{mL}$

$\mathrm{e}$

g
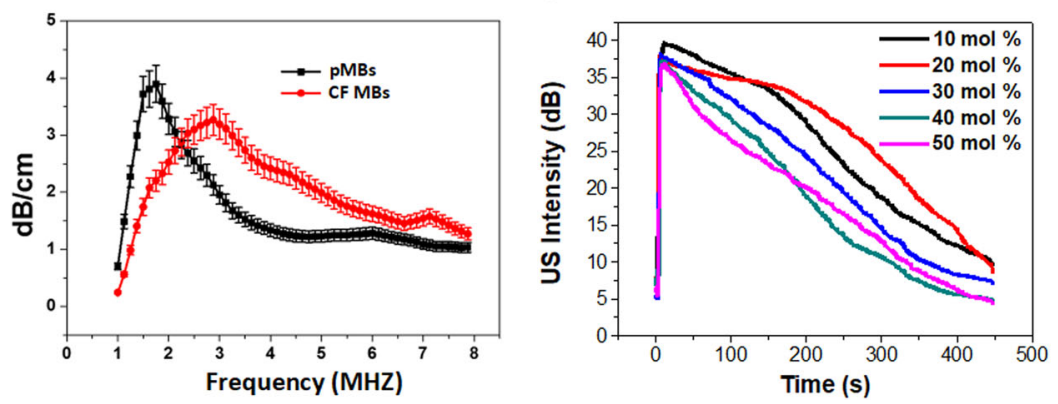

$\mathrm{h}$

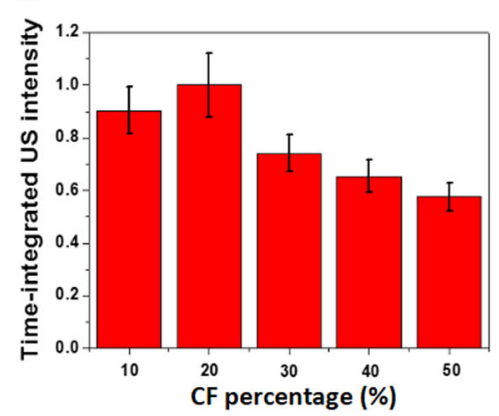

Fig. 1 Design and characterization of CF MBs. a $\pi-A$ isotherms for mixtures of CF/DSPC/DSPE-PEG2000 on the PBS subphase at pH 7.4 at different molar ratios of CF: 0 mol\%, 10 mol\%, 20 mol\%, 30 mol\%, 40 mol\%, 50 mol\% and 100 mol\%. b Microscopic images of representative CF MBs-30\%; the molar ratio of DSPC/DSPE-PEG2000 is fixed at 9:1 (scale bar $=20 \mu \mathrm{m}$ ). c In vitro US contrast-enhanced images of CF MBs-30\% with increasing concentrations in a latex tube. $\mathbf{d}$ Normalized US signal intensities in (c) over CF MBs-30\% concentration. e Acoustic attenuation as a function of frequency for pMBs without CF and CF MBs-30\%. f In vivo US imaging of CF MBs-30\% intravenously administered into SD rats. The kidney was imaged with a clinical US probe (3 12 MHz). g Time-lapse US signal intensity: CF MBs-10\%, CF MBs-20\%, CF MBs-30\%, CF MBs-40\%, and CF MBs-50\%. h Time-integrated US contrast enhancement of CF MBs- $10 \%$, CF MBs- $20 \%$, CF MBs-30\%, CF MBs- $40 \%$, and CF MBs- $50 \%$

then gradually decreased to pure CF-like values when the CF molar ratio exceeded $40 \mathrm{~mol} \%$. These results demonstrate that CF can form a stable film and that the incorporation of DSPC/DSPE-PEG2000 (9:1) can further improve the stability of the monolayer film. This finding indicated the possibility of generating $\mathrm{MBs}$ with $\mathrm{CF}$ by introducing DSPC/DSPE-PEG2000 (9:1) for further stabilization.

To overcome the limitation of the very low drug-loading capacity of conventional MBs, a new type of MB was engineered by encapsulating a perfluoropropane gas core with a CF shell using a mechanical agitation method. The phospholipids of DSPC/DSPE-PEG2000 (9:1) are introduced to improve the stability of the CF MBs. Then, the CF MBs were purified by centrifugation $\left(850 \mathrm{r} \mathrm{min}^{-1}\right.$ for $5 \mathrm{~min}$ ) to remove residual nanoparticles. Next, the MBs were washed with a mixed solvent containing glycerol, 1,2propyleneglycol, and ultrapure water ( $10 \mathrm{vol} \%: 10 \mathrm{vol}$ $\%: 80$ vol\%). Nevertheless, incorporating phospholipids may reduce the drug-loading contents of the CF MBs. To balance the stability and drug-loading contents of the $\mathrm{CF}$ $\mathrm{MBs}$, extensive efforts have been made to optimize the formulations by varying the molar ratios of $\mathrm{CF}$ and phospholipids. To determine the drug-loading contents of 
Table 1 Composition, concentration, mean diameter, and loading content of CF MBs

\begin{tabular}{lllll}
\hline Formulations & CF:DSPC:DSPE-PEG2000 (mol:mol:mol) & MB concentration $\left(\times 1 \mathbf{0}^{\mathbf{9}} \mathbf{M B s}\right.$ per $\left.\mathbf{~} \mathrm{mL}\right)$ & Mean diameter $(\boldsymbol{\mu m})$ & Loading content (\%) \\
\hline pMBs & $0: 90: 10$ & $5.60 \pm 0.34$ & $1.87 \pm 0.46$ & 0 \\
CF MBs-10\% & $10: 81: 9$ & $5.72 \pm 0.43$ & $1.31 \pm 0.39$ & $9.60 \pm 0.2 \%$ \\
CF MBs-20\% & $20: 72: 8$ & $8.10 \pm 0.48$ & $1.08 \pm 0.37$ & $25.40 \pm 1.1 \%$ \\
CF MBs-30\% & $30: 63: 7$ & $5.37 \pm 0.36$ & $0.95 \pm 0.29$ & $33.30 \pm 0.8 \%$ \\
CF MBs-40\% & $40: 54: 6$ & $4.44 \pm 0.39$ & $0.93 \pm 0.25$ & $45.02 \pm 1.5 \%$ \\
CF MBs-50\% & $50: 45: 5$ & $4.06 \pm 0.31$ & $0.91 \pm 0.36$ & $56.70 \pm 2.3 \%$ \\
\hline
\end{tabular}

CF in the MBs, the MBs were dissolved with dimethyl sulfoxide (DMSO), and the CF amount was determined by using the corresponding absorbance standard calibration curve (with a characteristic CPT peak at $361 \mathrm{~nm}$ ) (Fig. S1 in Supporting Information). All the newly generated $\mathrm{MB}$ suspensions were opaque and slowly delaminated (Fig. S2 in Supporting Information), confirming the effective formation of MBs. As shown in Table 1, all formulations showed good MB generation yields with concentrations of $\sim 10^{9}$ MBs per $\mathrm{mL}$. It was found that the CF molar percentage had an impact on the stability of the CF MBs. In particular, CF MB-20\% had the highest MB production yield with a concentration of $8.1 \times 10^{9} \mathrm{MBs}$ per $\mathrm{mL}$, but its drug-loading content was relatively low $(\sim 25.4 \%)$. Of particular interest, the incorporation of CF resulted in a decrease in the mean diameter compared with that of pMBs featuring a shell composed of DSPC/DSPEPEG2000 (9:1) and no CF. As the CF molar ratio increased from 0 to $50 \mathrm{~mol} \%$, the mean diameter of the CF MBs decreased from $1.87 \pm 0.46$ to $0.91 \pm 0.36 \mu \mathrm{m}$ (Table 1 , Fig. $1 \mathrm{~b}$ and Fig. S3, S4 in Supporting Information). CF is capable of forming a very condensed monolayer shell to impede gas escaping from the core into the aqueous medium, resulting in smaller MBs. In addition, the introduction of DSPE-PEG2000 into the MB shell prevents coalescence due to the bulky hydrophilic PEG headgroup pointing into the continuous media, thereby stabilizing the CF MBs. Furthermore, CF MBs were incubated in serum, and the $\mathrm{MB}$ concentration was measured at different incubation times. The results showed that the MB concentration slightly decreased at 5 min and further decreased to half of its concentration at $2.5 \mathrm{~h}$ (Fig. S5 in Supporting Information). Therefore, these CF MBs exhibit high stability.

\section{Echogenicity and US-triggered in situ micro-to-nano conversion of CF MBs}

To investigate the diagnostic potential of CF MBs as an US contrast agent, an in vitro experiment in a latex tube was performed first. A CF MBs-30\% suspension was injected into the latex tube, which contained circulating saline to simulate a blood vessel. As shown in Fig. 1c, for the PBS sample without CF MBs, no US signal could be detected. In contrast, when the CF MB concentration changed from 0 to $6 \times 10^{4} \mathrm{MBs}$ per $\mathrm{mL}$, the generated US signals gradually increased at lower concentrations, and the echo intensity was nearly saturated at $5 \times 10^{4} \mathrm{MBs}$ per $\mathrm{mL}$ (Fig. 1d), showing their excellent US contrast capability.

To characterize the linear acoustic behavior of CF MBs, the acoustic attenuation spectrum of CF MBs-30\% was measured. It was found that the acoustic attenuation spectrum was dependent on the acoustic frequency and the size distribution of the bubbles. The MBs without CF first displayed a sharp increase in attenuation from 1.0 to $1.5 \mathrm{MHz}$, exhibiting a peak value of $4.0 \mathrm{~dB} \mathrm{~cm}^{-1}$ at 1.75 $\mathrm{MHz}$, followed by a subsequent decline to $1.2 \mathrm{~dB} \mathrm{~cm}^{-1}$ at $8 \mathrm{MHz}$. In contrast, CF MBs-30\% with a narrower volumetric size distribution also produced a sharp rise in US attenuation at higher frequency, exhibiting a peak value of $3.1 \mathrm{~dB} \mathrm{~cm}^{-1}$ at approximately $3.25 \mathrm{MHz}$, and the attenuation gradually tapered off to $1.2 \mathrm{~dB} \mathrm{~cm}^{-1}$ at $8 \mathrm{MHz}$ (Fig. 1e). Thus, CF MBs having a higher resonance frequency at approximately $3.25 \mathrm{MHz}$ may be well suited for imaging applications in the $3-7 \mathrm{MHz}$ frequency range, which has been widely applied in the clinic.

We next examined the unique capability of CF MBs with different CF contents for in vivo US imaging. When CF MBs-30\% were intravenously injected into a rabbit, the signal from the renal cortex became remarkably enhanced in real time. CF MBs-30\% exhibited a strong echo signal, and the contrast-enhanced imaging lasted for more than $5 \mathrm{~min}$ (Fig. 1f). This significant harmonic property, which enhances grayscale imaging, permits the acquisition of dynamic blood flow information and visualization of normal organs or tumors with well-defined margins. Similar results were also observed for other CF MBs. The US intensity change in kidneys injected with CF MBs with different formulations was plotted with time (Fig. 1g). At $13 \mathrm{~s}$ after injection, the US signal rapidly reached its peak intensity for all CF MBs, while different attenuation trends were observed for the different CF MBs. The timeintegrated US intensities were further normalized (Fig. 1h), showing the order of CF MBs-20\% > CF MBs- 
a
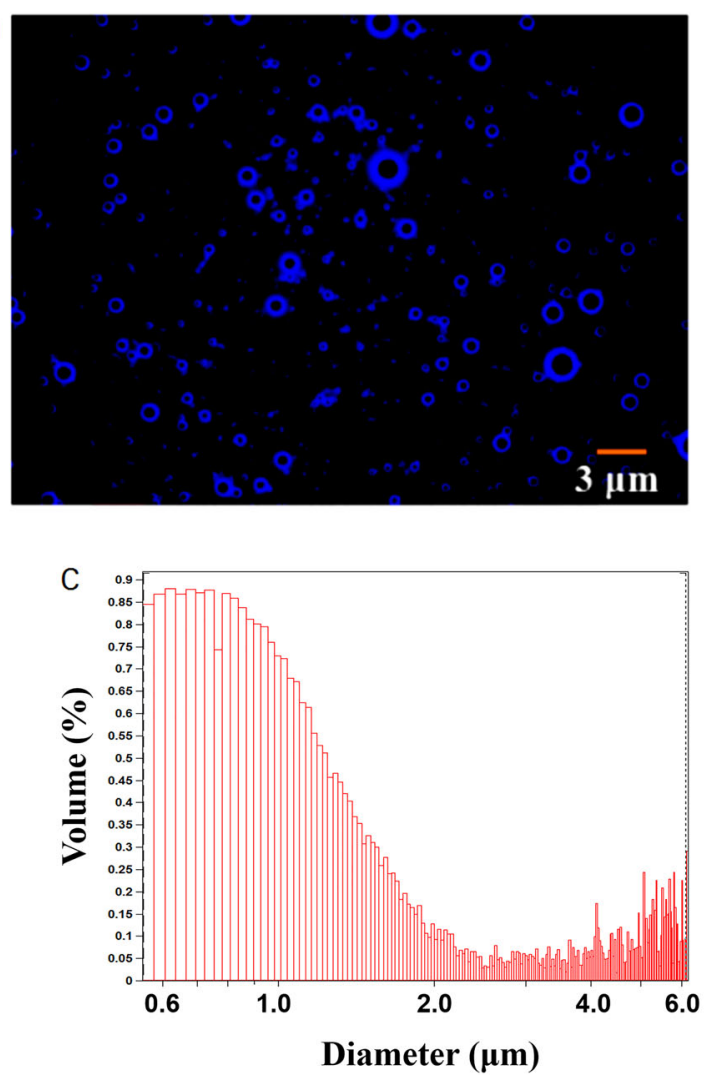

b
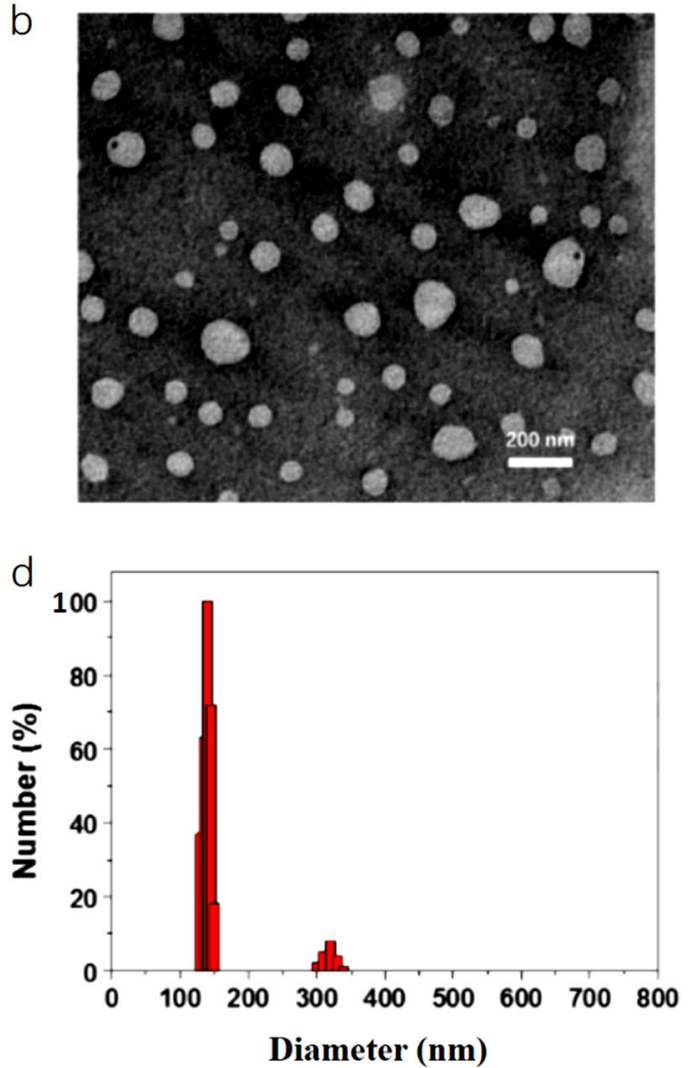

Fig. 2 US-induced conversion of CF MBs to CF NPs. a Fluorescence microscopy image of CF MBs, where blue fluorescence was generated from CF. $\mathbf{b}$ TEM image of CF NPs. c Size distribution of CF MBs, and $\mathbf{d}$ size distribution of the CF NPs converted from CF MBs upon US exposure

$10 \%>$ CF $\mathrm{MBs}-30 \%$ > CF MBs- $40 \%$ > CF MBs- $50 \%$. This result is consistent with the collapse pressure results of the different CF formulations (Fig. 1a). To balance stability and drug-loading content, CF MBs-30\% were chosen for all the following studies.

To explore whether CF MBs can be converted into nanoparticles, CF MBs were exposed to US $(1.03 \mathrm{MHz}$, $50 \%$ high-duty-cycle, $1 \mathrm{~W} \mathrm{~cm}^{-2}$ ) for $1 \mathrm{~min}$. As shown in Fig. 2a, c, before US exposure, the fluorescence images showed the formation of spherical MBs ranging from 0.6 to $1.2 \mu \mathrm{m}$ in size, and the peak size was approximately 0.9 $\mu \mathrm{m}$. After US exposure, the transmission electron microscopy (TEM) images showed spherical nanoparticles ranging from 70 to $200 \mathrm{~nm}$ in size (Fig. 2b), which is consistent with the hydrodynamic light scattering measurements $(\sim 150 \mathrm{~nm})$ (Fig. 2d and Fig. S6 in Supporting Information). This result verified that CF MBs could be efficiently converted into CF NPs upon US exposure.

Enhancing the cellular uptake and cytotoxicity of CF MBs with the aid of US

To study the cellular uptake of CF MBs after UStriggered conversion into nanoparticles, the US parameter for in vitro experiments was optimized. US irradiation was found to cause no cytotoxicity when the sound intensity was below $1.5 \mathrm{~W} \mathrm{~cm}^{-2}$ (Fig. S7a in Supporting Information). In particular, a sound intensity of $1.0 \mathrm{~W} \mathrm{~cm}^{-2}$ was the most beneficial to cellular uptake, as it led to the strongest fluorescence of CF being emitted from cells (Fig. S7c in Supporting Information) and caused almost no cytotoxicity resulting from the cavitation effect when the concentration of phospholipid MBs (pMBs) reached $20 \mu \mathrm{M}$ (Fig. S7b in Supporting Information).

Cellular uptake of CF MBs with or without US exposure was studied by fluorescence microscopy and flow cytometry. The CPT fluorescence of CF molecules was used to detect cellular uptake without using other additional markers. The data showed that the CF MBs were actively taken up by HepG2 cells in a timedependent manner, which is likely because the CF MBs were broken into CF NPs during incubation with the tumor cells, thus showing similar uptake behavior to the CF NPs (Fig. S8 in Supporting Information). Nevertheless, CF MB-treated cells exhibited obviously increased fluorescence upon US exposure, and the outstanding colocalization of CF (in blue) and nuclei (stained with propidium iodide, in red) showed the 


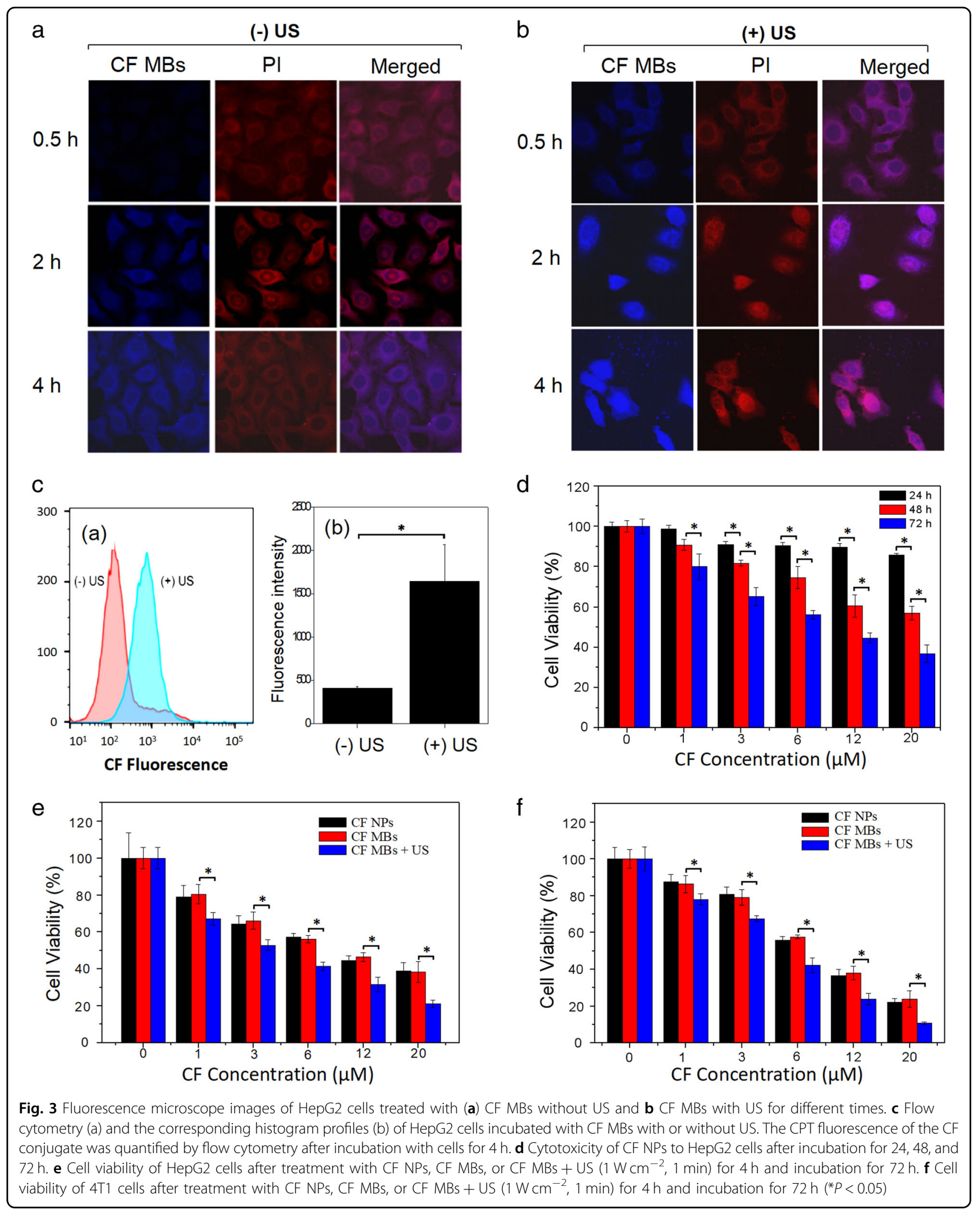


strong uptake of the CF NPs (Fig. 3a, b), suggesting that CF MB + US-induced sonoporation can greatly enhance cell membrane permeability. In addition, the fluorescence of CPT was visualized in both the cell cytoplasm and nuclei, indicating that CF MBs + US caused in situ conversion of CF MBs into smaller CF NPs that penetrated into the cells due to the CF MBs + US-induced cavitation. Then, the CF molecules were hydrolyzed to

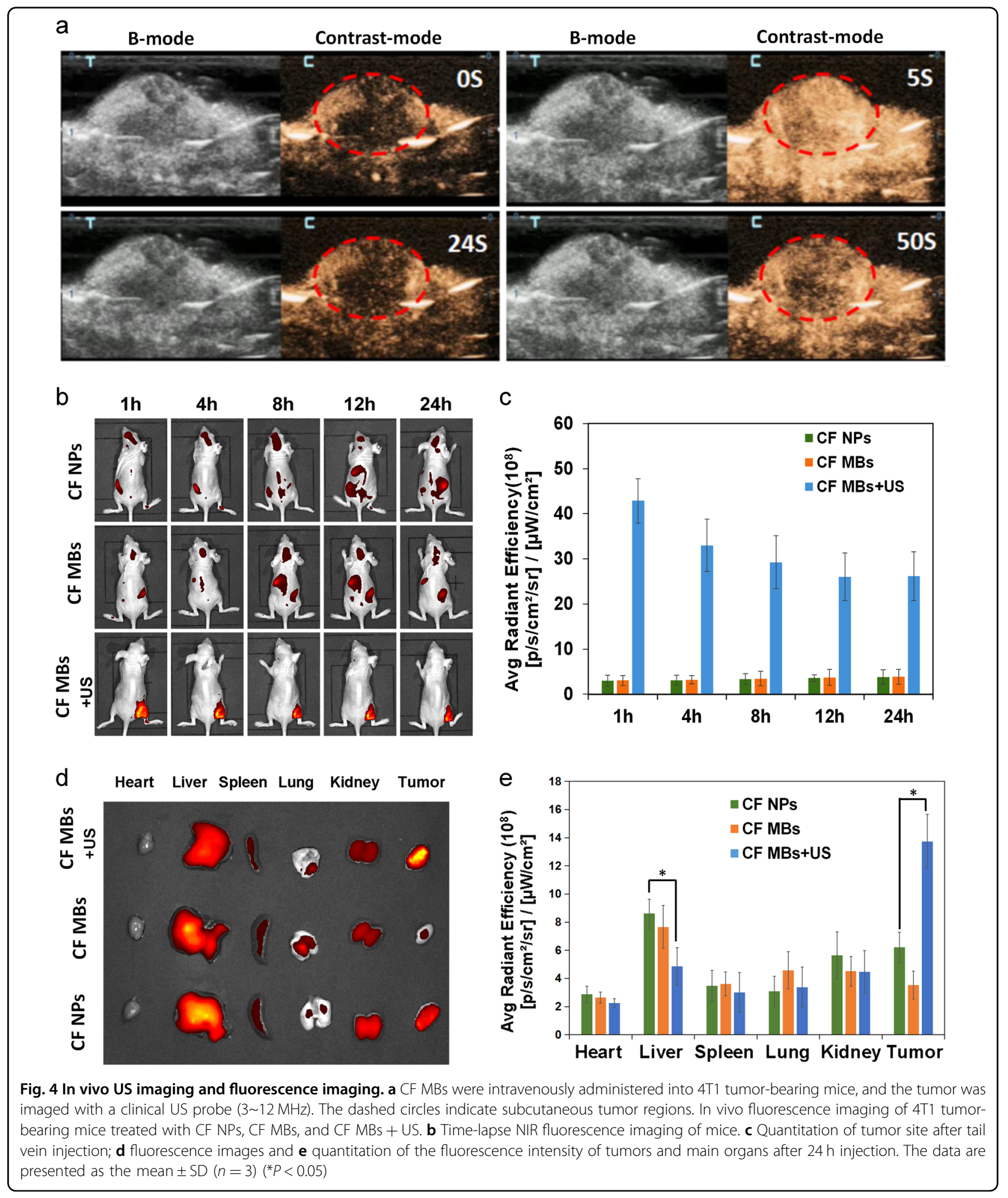


release CPT and FUDR molecules in the microenvironment of the tumor cells. Compared to that in the group without US exposure, the fluorescence in the CF $\mathrm{MB}+\mathrm{US}$ group was increased by 3.48 -fold due to the effect of sonoporation (Fig. 3c). The significantly increased cellular uptake demonstrated that CF MBs combined with US can be a powerful strategy for improving drug delivery efficiency.

The in vitro cytotoxicity of CF MBs to cancer cells was evaluated by the MTT assay with CF NPs and CPT\&FUDR@liposome (CPT and FUDR-loaded liposome) as controls. The CF NPs exhibited obvious cytotoxicity to HepG2 cells when the incubation time was prolonged to $72 \mathrm{~h}$, indicating that the free drugs were sustainably released from CF owing to the esterase- and acid-mediated hydrolysis of the ester bond of CF in the tumor cells (Fig. 3d). Compared with the release ratio of the free drugs from CPT\&FUDR@liposome, the release ratio of the free drugs from CF NPs was slower, as almost all free drugs inside the liposomes were released within $24 \mathrm{~h}$, while it took $72 \mathrm{~h}$ for the CF NPs to cause the same cytotoxic effect (Fig. S9 in Supporting Information and Fig. 3d). For both CF NP- and CF MB-treated HepG2 cells, the cytotoxicity was similar and concentration dependent. Nevertheless, CF MBs combined with US resulted in a greater inhibitory effect on HepG2 cancer cell growth (Fig. 3e). This result should be attributed to the fact that CF MBs could be converted into small CF NPs with the assistance of US irradiation, which further enhanced their cellular internalization due to the USinduced sonoporation effect. Similar results were also obtained for 4T1 tumor cells after various treatments (Fig. 3f). Therefore, CF MBs + US can greatly improve drug delivery efficiency and therapeutic cytotoxicity, which is consistent with the results of the cellular uptake experiments (Fig. 3a-c).

\section{Enhanced drug tumor accumulation by US-targeted destruction of CF MBs}

Contrast-enhanced US imaging with a clinical US L12$3 \mathrm{U}$ probe $(3 \sim 12 \mathrm{MHz})$ was utilized to monitor the influx of $\mathrm{CF}$ MBs into the tumor tissue by intravenously injecting CF MBs into 4T1 tumor-bearing mice. Before injection $(0 \mathrm{~s})$, there was almost no US contrast signal in the tumor. At $5 \mathrm{~s}$ postinjection, the strongest backscatter signals were observed in the tumor tissue, demonstrating that the CF MBs flowed into the tumor (Fig. 4a and Fig. S10 in Supporting Information). After $24 \mathrm{~s}$ postinjection, low-frequency US was exposed to the tumor for in situ conversion of CF MBs into nanoparticles, leading to an instantaneous decrease in the US contrast signal. After $10 \mathrm{~s}$, the CF MBs gradually reperfused the tumor, partially recovering the US contrast signal. After approximately $3 \mathrm{~min}$, there was negligible backscatter signal in the tumor. It was impressive that CF MBs can act as an effective US contrast agent not only to enhance tumor imaging but also to respond to low-frequency US for conversion into nanoparticles. This finding prompted us to investigate the use of CF MBs for US-mediated local drug delivery in vivo.

The efficient cellular uptake and significant inhibitory effect of CF MBs combined with US in tumor cells motivated us to investigate their in vivo biodistribution. A small amount of a near-infrared florescence dye, porphyrin-conjugated lipid (PGL, $1.0 \mathrm{~mol} \%)^{35}$, was incorporated into the CF MBs (or CF NPs) to track the therapeutic agents by fluorescence imaging. A whole-body NIR imaging approach was utilized to investigate the tumor accumulation of CF MBs combined with US in nude mice. Figure $4 \mathrm{~b}$, c shows the fluorescence signal distributions as a function of time for CF NPs, CF MBs, and $\mathrm{CF}$ MBs + US delivered systemically via tail vein injection. For the group treated with CF MBs + US, the tumor site showed an apparent fluorescence signal at $1 \mathrm{~h}$ postinjection, and the signal at the tumor was then retained for the following $23 \mathrm{~h}$ with little signal decrease. In sharp contrast, the fluorescence signal of the CF NPand $\mathrm{CF}$ MB-treated groups was very weak in the tumor but very strong in the liver within $24 \mathrm{~h}$ of observation. The $\mathrm{CF} \mathrm{MB}+\mathrm{US}$ group showed a 14 times higher fluorescence signal in the tumor tissue than the CF NP- and CF MB-treated groups (Fig. 4c). This provides strong evidence that CF MBs combined with US can not only significantly enhance drug accumulation at the tumor sites but also remarkably reduce the drug concentration in organs such as the liver and spleen due to the enhanced vasculature permeation and cellular uptake enabled by the sonoporation effect.

At $24 \mathrm{~h}$ postinjection, we further removed the major organs and quantified the fluorescence intensity to evaluate the biodistribution of the CF NPs, CF MBs, and CF $\mathrm{MBs}+$ US (Fig. 4d, e). Semiquantitative analysis of various tissues showed very strong fluorescence originating from the liver, which was due to the macrophage clearance of the nanoparticles from the blood. The tumor fluorescence intensity of the CF MBs + US was remarkably higher than that of the CF NPs and CF MBs. Based on the above results, it was reasonable to argue that US-targeted CF MB destruction can enhance the tumor-specific uptake of the in situ converted NPs, surpassing the EPR effect in vivo. In conventional chemotherapy, the maximum tolerated dose of a drug is usually applied to achieve the highest tumor cell uptake, resulting in the largest therapeutic efficacy while causing serious side effects. Therefore, CF MBs combined with US were crucial to overcome the heterogeneity of the tumor microenvironment and efficiently accumulated in the tumor tissue without premature release, showing great potential for 
achieving improved therapeutic effects and reduced side effects.

\section{Enhancing therapeutic efficacy by combining CF MBs and US}

To evaluate the in vivo antitumor efficacy of CF MBs, mice bearing 4T1 tumors with initial tumor volumes of approximately $100 \mathrm{~mm}^{3}$ were randomly divided into five groups: (a) PBS, (b) CPT\&FUDR@liposome, (c) CF NPs, (d) CF MBs, (e) CF MBs + US. Equivalent drug doses of all of the therapeutic agents were intravenously injected via the tail vein, and the tumor volumes or body weights were recorded every day after the different treatments (Fig. 5a, b). The in vivo antitumor results showed that the size of the tumors in group (e) treated with CF MBs + US increased much slower than that of the tumors in the other groups. At 14 days after treatment with (a) PBS, (b) CPT\&FUDR@liposome, (c) CF NPs, or (d) CF MBs, the tumor volume increased to approximately 1850, 1450, 850 , and $800 \mathrm{~mm}^{3}$, respectively. At the same injected drug dose, the CF NPs and CF MBs inhibited tumor growth more effectively than the CPT\&FUDR@liposome (Fig. 5a, c). The structure of the Janus camptothecin-floxuridine conjugate prevented the premature release of camptothecin or floxuridine, resulting in improved antitumor efficacy of the CF NPs or CF MBs. In marked contrast, the smallest tumor volume was observed in group (e) treated with CF MBs + US (approximately $510 \mathrm{~mm}^{3}$ ) under the same experimental conditions. The significantly enhanced antitumor efficacy was consistent with the biodistribution results. By using the UTMD technique, CF MBs with high drug-loading contents were converted into CF NPs and effectively accumulated in tumors due to the enhanced vasculature permeation and cellular uptake enabled by the sonoporation effect, circumventing the limitations of the EPR effect. Moreover, the lack of time- (Fig. 5b) or organ damage (Fig. S11 in Supporting Information)-dependent body weight loss suggested that none of these various therapeutic agents had significant toxicity in the animal experiments.

Histological examination of tumor apoptosis and necrosis was performed by hematoxylin-eosin staining (H\&E) and immunohistochemical analysis for Ki67 (Fig. 5d). For H\&E staining, the CF MBs + US-treated group exhibited the most serious nuclear deficiency among the five groups. Immunohistochemical analysis showed that Ki67 expression, a cellular marker exclusively linked with cell proliferation, was considerably reduced in the group treated with CF MBs + US compared with the other groups. The superior antitumor efficacy of CF MBs + US facilitated by inducing apoptosis or necrosis in 4T1 tumors should be attributed to the synergistic effect of $\mathrm{CPT} / \mathrm{FUDR}$ and the enhanced intracellular uptake of CF induced by US.

\section{Conclusions}

In summary, we successfully engineered CF MBs with ultrahigh loading contents (up to 56.7\%) of two synergetic drugs and an excellent capability to enhance US imaging for identifying the location and size of tumors. In addition, CF MBs could be efficiently converted into CF NPs by bursting the MBs with a US beam under the guidance of US imaging, enabling remarkably high drug tumor accumulation via the sonoporation effect. CPT and FUDR could be released at an exact 1:1 ratio in the tumor microenvironment, achieving coordinated pharmacokinetics. The increased selectivity of the treatment with CF MBs combined with US resulted in significantly improved therapeutic efficacy and reduced undesirable side effects. Further studies using targeting ligands for modification of CF MBs to further increase the selectivity are merited. In summary, CF MBs combined with US offer a powerful strategy to optimize the action of CPT and FUDR. Furthermore, CF MBs can act as carriers to load other drug substances (such as plasmid DNA) by attachment to the surface or incorporation into the shell of the CF MBs until the site of interest is reached, as they are sufficiently stable for circulation in the vasculature as blood pool agents. Overall, the CF MBs combined with US provides a step toward the application of targeted personalized diagnostics and therapy.

\section{Experimental section Materials}

CF was synthesized by conjugating two hydrophobic CPT molecules and two hydrophilic FUDR molecules to multivalent pentaerythritol by a hydrolyzable ester linkage. The synthesis was achieved using a protection -deprotection strategy due to its four symmetric and highly reactive hydroxyl groups according to our reported method $^{33-35}$. Camptothecin and floxuridine were purchased from TCI (Shanghai, China). Dipalmitoyl-sn-glycero-3-phosphocholine (DSPC) and 1,2-distearoyl-snglycero-3-phosphoethanolamine- $N$-[methoxy(polyethylene glycol)-2000] (DSPE-PEG2000) were obtained from Shanghai A.V.T. Pharmaceutical Co., Ltd. All other reagents and solvents were purchased from domestic suppliers and used as received.

\section{Langmuir monolayers}

A spreading film was prepared by dropping a chloroform solution $\left(50 \mu \mathrm{L}, 1 \times 10^{-4} \mathrm{M}\right)$ of $\mathrm{CF}$ onto an aqueous surface (PBS subphase at $\mathrm{pH} 7.4$ ) with a gastight Hamilton microsyringe. The $\pi-\mathrm{A}$ isotherms were recorded on a KSV 1100 minitrough (KSV instruments 1100, Helsinki, Finland) at $20^{\circ} \mathrm{C}$ by compressing the film at a rate of 5 $\mathrm{mm} \mathrm{min}{ }^{-1}$ after allowing the chloroform to evaporate for $20 \mathrm{~min}$. 

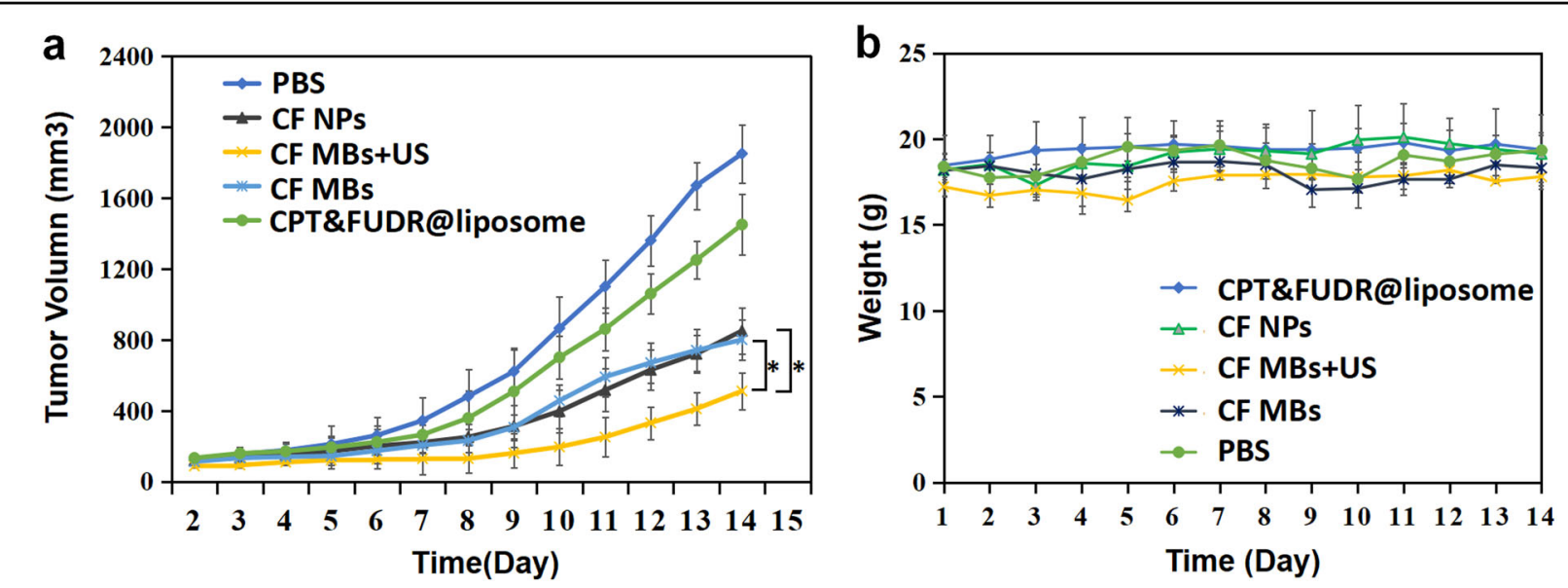

C
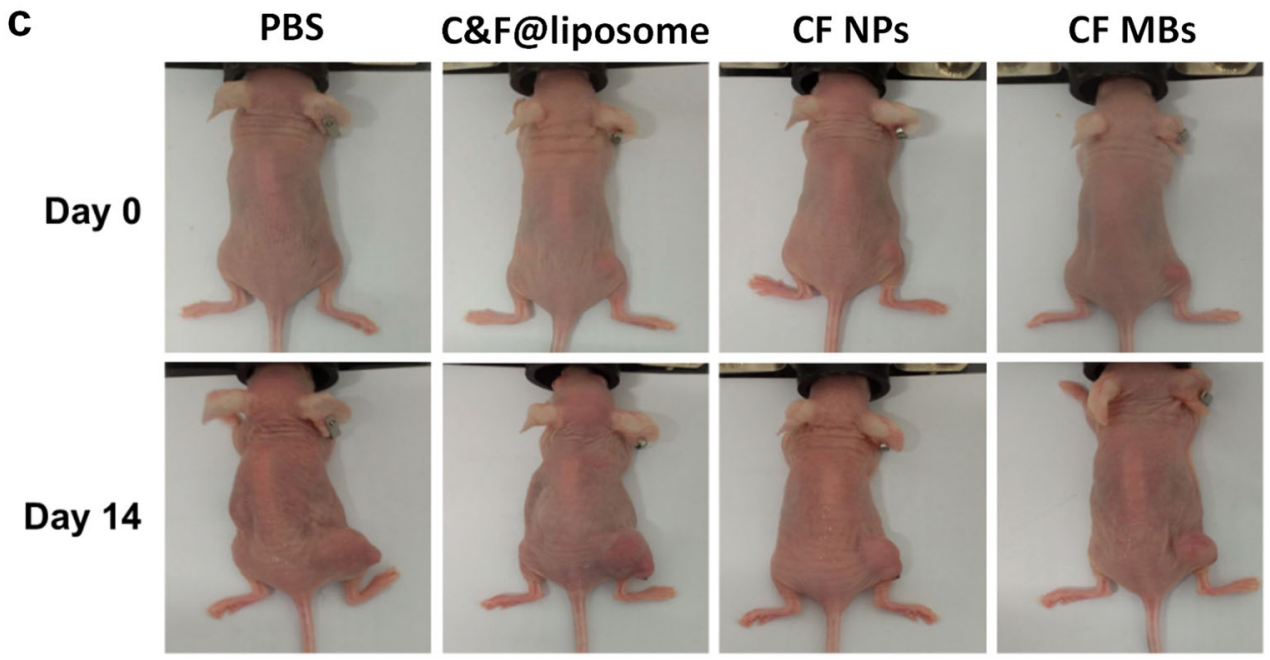

CF MBs+US

d

\section{PBS}
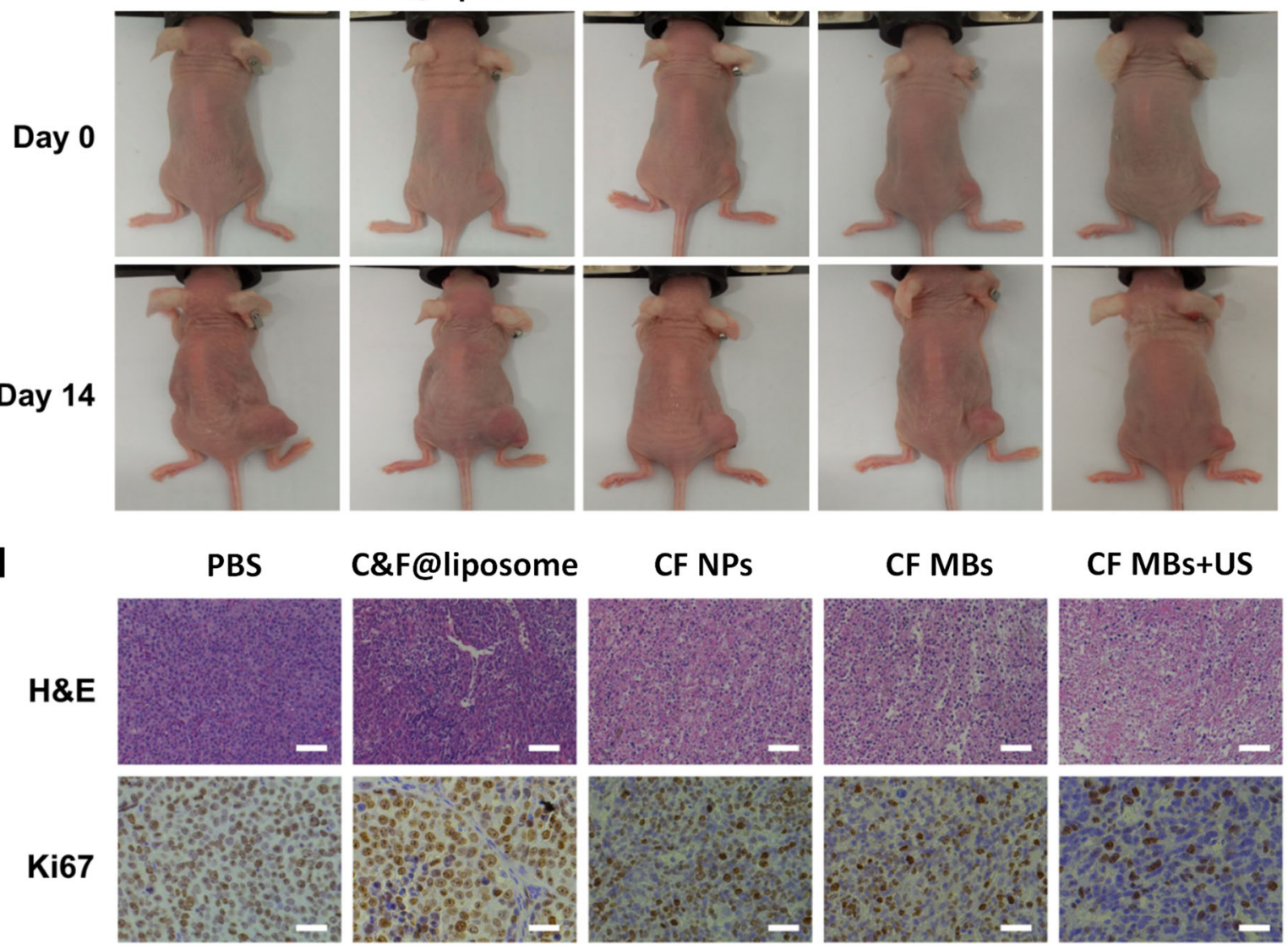

CF NPs

CF MBs

CF MBs+US
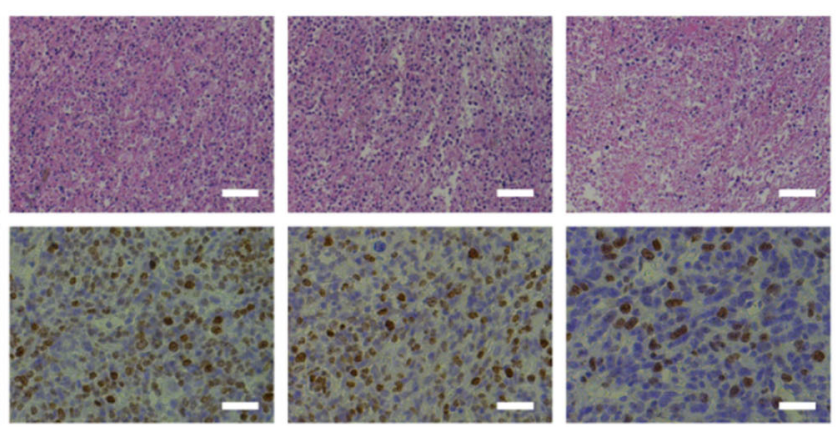

Fig. 5 In vivo antitumor effect of CF MBs combined with US on 4T1 tumor-bearing nude mice. a Tumor volume change over time after treatment with CPT\&FUDR@liposome, CF NPs, CF MBs and CF MBs + US, ${ }^{*} P<0.05$. b Changes in body weight over time. Data are shown as the mean \pm standard deviation (SD), $n=5$. c Images of tumor-bearing mice after different treatments. $\mathbf{d}$ Tumors were sectioned and stained with hematoxylin and eosin or subjected to immunohistochemical evaluation of Ki67 after different treatments. Scale bar $=40 \mu \mathrm{m}$

\section{Fabrication of CF MBs}

CF NPs were first self-assembled by dissolving $1.5 \mathrm{mg}$ of the mixture of DSPE-PEG2000, DSPC and CF with different molar ratios in $200 \mu \mathrm{L}$ of DMSO, followed by rapid injection into $1 \mathrm{~mL}$ of deionized water under water bath sonication to obtain a homogeneous and opalescent suspension. Afterwards, the suspension was further homogenized by probe sonication for $10 \mathrm{~min}$ (Sonicator 4000, Misonix) at $30 \%$ output amplitude setting in an ice-water bath. Finally, the resulting solution was dialyzed (MWCO 
8000-14000) against deionized water to completely remove DMSO. The obtained CF NP suspension was then transferred into a small sample vial, and the air above the solution was replaced with perfluoropropane $\left(\mathrm{C}_{3} \mathrm{~F}_{8}\right)$ gas. Finally, the sample was mechanically agitated for $45 \mathrm{~s}$ using a VialMix shaker to obtain the CF MBs, which were further purified by centrifugation at $850 \mathrm{rpm}$ for $5 \mathrm{~min}$.

\section{Physical characterization}

Optical observations of MBs were carried out with a Leica DM 4500P microscope attached to a Leica DFC 420 digital camera. The size distribution and concentration of the MBs were determined on diluted samples in isotonic solution using a Coulter Multisizer III (Coulter Electronics Ltd., Luton, Bedfordshire, UK) with a $30 \mu \mathrm{m}$ aperture, a $100 \mu \mathrm{L}$ manometer setting and aperture current of 1600 $\mu \mathrm{A}$. The sample was normalized to a final concentration of $1.0 \times 10^{9}$ bubbles $\mathrm{mL}^{-1}$ (using PBS) for the in vivo acoustic test. Just prior to use, the glass vials were gently rotated to evenly distribute the bubbles.

The characteristic structure of the CF NPs was investigated by TEM using an FEI Tecnai T20 transmission electron microscope operated at an accelerating voltage of $200 \mathrm{kV}$. The samples were stained with phosphotungstic acid (3wt\%) before TEM imaging. The size distributions and zeta potentials of the CF NPs were evaluated using a 90Plus/BI-MAS instrument (Brookhaven Instruments Co., U.S.). UV/vis absorption spectra were measured by a UV-vis spectrophotometer (Thermo Fisher Scientific Evolution 220). Fluorescence spectra were recorded using a fluorescence spectrophotometer (Thermo Fisher Scientific Lumina).

\section{CF MBs acoustic measurements}

Frequency-dependent attenuation measurements were performed to assess the response of the CF MBs. A narrowband pulse-echo approach was employed: two transducers (model \#595396, $2.25 \mathrm{MHz}, 76 \mathrm{~mm}$ focus, $12.7 \mathrm{~mm}$ diameter; Olympus NDT Canada Inc., Quebec, Canada; model \#ISO2002HR, $5 \mathrm{MHz}, 38 \mathrm{~mm}$ focus, $6.35 \mathrm{~mm}$ diameter; Valpey-Fisher, Hopkinton, MA, USA) were used to cover a frequency range of $1.0-8 \mathrm{MHz}$. The transducers were situated within a water bath, and their beams passed through a sample chamber containing diluted agent via a Mylar window and were focused on aluminum reflectors. An arbitrary waveform generator (model AWG5002C; Tektronix, Beaverton, OR, USA) was used to generate low-amplitude, narrowband pulses. The input waveforms were amplified by $53 \mathrm{~dB}$ (model-150; ENI, Rochester, NY, USA) and then sent to one of the two transducers. Upon receipt, the echoes were amplified by $35 \mathrm{~dB}$ (model AU1583; Miteq, Hauppauge, NY, USA), bandpass filtered and then digitized (400 MHz sampling frequency; Agilent Technologies Inc., Palo Alto, CA, USA) for offline analysis. The resulting echo at each frequency was averaged ten times for signal-to-noise ratio purposes. Experiments were conducted with agent diluted (1:15000) in saline $(0.9 \% \mathrm{NaCl})$, and acoustic measurements commenced at the $1 \mathrm{~min}$ mark following dilution. Experiments were repeated twice per vial using three vials for each type of MB.

\section{Cellular uptake of CF MBs}

For confocal laser scanning microscopy, human liver cancer HepG2 cells were cultured in 24 -well dishes $\left(1 \times 10^{5}\right.$ cells/well) with $2 \mathrm{~mL}$ of complete DMEM. After incubation for $24 \mathrm{~h}$, the culture media were replaced with $0.5 \mathrm{~mL}$ of fresh medium containing CF MBs or CF MBs followed by US (unfocused $1.03 \mathrm{MHz}$ US at a $1 \mathrm{~W} \mathrm{~cm}^{-2}$ power density and $50 \%$ duty cycle for $1 \mathrm{~min}$ ). Afterwards, the cells were cultivated for a prearranged time $(0.5,2,4 \mathrm{~h})$ at $37^{\circ} \mathrm{C}$. Following the removal of culture media, the cells were washed with PBS and then fixed with $70 \%$ ethanol for 30 $\min$ at $4{ }^{\circ} \mathrm{C}$. Subsequently, the cells were stained with propidium iodide (PI, $1 \mu \mathrm{g} \mathrm{mL}^{-1}$ ) for $5 \mathrm{~min}$ to visualize the nucleus. Finally, the slides were rinsed with PBS and then mounted with antifade solution. The resulting slides were imaged with a LEICA TCS SP8 fluorescence microscope.

For flow cytometry, the HepG2 cells were plated in sixwell dishes $\left(5.0 \times 10^{5}\right.$ cells/well $)$ with $2 \mathrm{~mL}$ of complete DMEM and allowed to adhere for $24 \mathrm{~h}$. The culture medium was subsequently removed, and fresh medium containing CF MBs was added. The cells were exposed to US or not and then incubated at $37^{\circ} \mathrm{C}$ for $4 \mathrm{~h}$. The cells were washed with PBS and detached via trypsinization. After preparation for flow cytometry analysis, data were acquired by using a BD LSRFortessa flow cytometer. A total of $1.0 \times 10^{4}$ gated events per sample were recorded.

\section{In vitro antitumor efficacy of CF MBs with or without US exposure}

For in vitro chemotherapy, HepG2 or 4T1 cells preseeded in 96 -well plates at a density of $1.0 \times 10^{4}$ per well were incubated with different drug formulations, followed by US exposure or not $\left(1.03 \mathrm{MHz}\right.$ US at a $1 \mathrm{~W} \mathrm{~m}^{-2}$ power density and $50 \%$ duty cycle for $1 \mathrm{~min}$ ) and incubation for $4 \mathrm{~h}$. The incubation media were then replaced with $2 \mathrm{~mL}$ of fresh complete media, followed by another $72 \mathrm{~h}$ of incubation. An MTT test was performed to evaluate the relative viability of the cells.

\section{In vivo US imaging}

$4 \mathrm{~T} 1$ breast cancer cells $\left(1.0 \times 10^{7}\right.$ in $50 \mathrm{~mL}$ PBS) were subcutaneously injected into the right hind leg of female $\mathrm{Balb} / \mathrm{C}$ mice, which were obtained from Beijing Weitonglihua Technology Co., Ltd. After 10 days, the tumor volume reached approximately $100 \mathrm{~mm}^{3}$. After the tumor-bearing mice were intravenously injected with 200 
$\mu \mathrm{L}$ of CF MBs $\left(2.0 \times 10^{8}\right.$ per $\left.\mathrm{mL}\right)$, the tumor was imaged using a Mindray Resona 7 US system with an L12-3U probe $(3 \sim 12 \mathrm{MHz})$ at a mechanical index (MI) of 0.04 . At $24 \mathrm{~s}$ postinjection, the MBs in the tumor were exposed to low-frequency US to observe the reinflux of the MBs into the tumor.

\section{In vivo fluorescence imaging and distribution analysis}

A near-infrared fluorescence dye, porphyrin-conjugated lipid (PGL) $)^{35}$, was incorporated into CF NPs or CF MBs for in vivo fluorescence imaging and for investigating the tumor-targeting capability of CF NPs or CF MBs (molar ratio of PGL: $1 \%$ ). Mice bearing 4T1 tumors were treated with $200 \mu \mathrm{L}$ of CPT\&FUDR@liposome, CF NPs, CF MBs or CF MBs + US $\left(1.03 \mathrm{MHz}, 50 \%\right.$ duty cycle, $1 \mathrm{~W} \mathrm{~cm} \mathrm{~cm}^{-2}, 3$ $\mathrm{min})$. Then, the mice were scanned with an IVIS Spectrum fluorescent imaging system (Lumina III) to collect the fluorescence signal of PGL at 1, 4, 8, 12, and 24h. At $24 \mathrm{~h}$ after fluorescence imaging, the mice were sacrificed to collect the heart, liver, spleen, lung, kidneys and tumor for semiquantitative analysis of biodistribution.

\section{In vivo antitumor efficacy of combination therapy}

Mice bearing 4T1 tumors were randomly divided into five groups ( $n=5$ per group). When the tumor volume reached approximately $100 \mathrm{~mm}^{3}$, the five groups of mice were treated with $200 \mu \mathrm{L}$ of (a) PBS, (b) CPT\&FUDR@liposome, (c) CF NPs, (d) CF MBs, or (e) CF MBs + US (1.03 MHz, 50\% duty, $1 \mathrm{~W} \mathrm{~cm}^{-2}, 3 \mathrm{~min}$ ) on the first day and $100 \mu \mathrm{L}$ of $(\mathrm{a}-\mathrm{e})$ on the fourth and sixth day (the concentration of the CF NPs or CF MBs was $1 \mathrm{mg} \mathrm{mL}^{-1}$ ). After various treatments, the tumor sizes and body weights were recorded every day for 14 days. The tumor volume was calculated by the following formula: length $x$ width $^{2} / 2$.

\section{Statistical analysis}

Student's $t$ test was applied to test the significance of the differences between two groups, and differences were considered significant when ${ }^{*} P<0.05$. Data were expressed as the mean \pm standard deviation $(\mathrm{SD})$.

\section{Data availability}

The authors declare that all data supporting the findings of this study are available within the paper and its Supplementary Information.

\section{Acknowledgements}

This research was financially supported by the National Key Research and Development Program of China (No. 2016YFA0201400), the National Project for Research and Development of Major Scientific Instruments (No. 81727803), the National Natural Science Foundation of China (Nos. 81771846 and 81571810), the Beijing Natural Science Foundation-Haidian Original Innovation Joint Fund (No. 17L20170), the Foundation for Innovative Research Groups of the National Natural Science Foundation of China (No. 81421004) and a grant from Peking University Third Hospital (BYSY2015023).

\section{Authors' contributions}

Z.D. designed the project. Z.D., X.L., Y.X., C.G., Y.Z., and N.Z. designed the experiment and wrote the manuscript. X.L. and C.G. fabricated the CF MBs, Y.Z. performed the cell experiment, Y.X. and N.Z. carried out the animal experiment. All authors analyzed the experimental data.

\section{Conflict of interest}

The authors declare that they have no conflict of interest.

\section{Publisher's note}

Springer Nature remains neutral with regard to jurisdictional claims in published maps and institutional affiliations.

Supplementary information is available for this paper at https://doi.org/ 10.1038/s41427-018-0066-x.

Received: 17 April 2018 Revised: 30 May 2018 Accepted: 10 June 2018. Published online: 17 August 2018

\section{References}

1. Maeda, H., Wu, J., Sawa, T., Matsumura, Y. \& Hori, K. Tumor vascular permeability and the EPR effect in macromolecular therapeutics: a review. J. Control. Release 65, 271-284 (2000).

2. Bertrand, N., Wu, J., Xu, X., Kamaly, N. \& Farokhzad, O. C. Cancer nanotechnology: the impact of passive and active targeting in the era of modern cancer biology. Adv. Drug Deliv. Rev. 66, 2-25 (2014).

3. Maeda, $\mathrm{H}$. The enhanced permeability and retention (EPR) effect in tumor vasculature: the key role of tumor-selective macromolecular drug targeting. Adv. Enzym. Regul. 41, 189-207 (2001).

4. Maeda, H. Toward a full understanding of the EPR effect in primary and metastatic tumors as well as issues related to its heterogeneity. Adv. Drug Deliv. Rev. 91, 3-6 (2015).

5. Ruoslahti, E., Bhatia, S. N. \& Sailor, M. J. Targeting of drugs and nanoparticles to tumors. J. Cell Biol. 188, 759-768 (2010)

6. Ernsting, M. J., Murakami, M., Roy, A. \& Li, S. D. Factors controlling the pharmacokinetics, biodistribution and intratumoral penetration of nanoparticles. J. Control. Release 172, $782-794$ (2013).

7. Dawidczyk, C. M. et al. State-of-the-art in design rules for drug delivery platforms: lessons learned from FDA-approved nanomedicines. J. Control. Release 187, 133-144 (2014).

8. Davis, M. E., Chen, Z. G. \& Shin, D. M. Nanoparticle therapeutics: an emerging treatment modality for cancer. Nat. Rev. Drug Discov. 7, 771-782 (2008).

9. Jain, R. K. Transport of molecules, particles, and cells in solid tumors. Annu. Rev. Biomed. Eng. 1, 241-263 (1999).

10. Heldin, C. H., Rubin, K, Pietras, K. \& Ostman, A. High interstitial fluid pressure an obstacle in cancer therapy. Nat. Rev. Cancer 4, 806-813 (2004).

11. Park, K. Facing the truth about nanotechnology in drug delivery. ACS Nano 7, 7442-7447 (2013).

12. Wilhelm, S. et al. Analysis of nanoparticle delivery to tumours. Nat. Rev. Mater. 1, 16014 (2016).

13. Lammers, T., Kiessling, F., Hennink, W. E. \& Storm, G. Drug targeting to tumors: principles, pitfalls and (pre-) clinical progress. J. Control. Release 161, 175-187 (2012).

14. Boissenot, T., Bordat, A., Fattal, E. \& Tsapis, N. Ultrasound-triggered drug delivery for cancer treatment using drug delivery systems: from theoretical considerations to practical applications. J. Control Release 241, 144-163 (2016).

15. Au, J. L., Yeung, B. Z., Wienties, M. G., Lu, Z. \& Wienties, M. G. Delivery of cancer therapeutics to extracellular and intracellular targets: determinants, barriers, challenges and opportunities. Adv. Drug Deliv. Rev. 97, 280-301 (2016).

16. Tzu-Yin, W., Wilson, K. E., Machtaler, S. \& Willmann, J. K. Ultrasound and microbubble guided drug delivery: mechanistic understanding and clinical implications. Curr. Pharm. Biotechnol. 14, 743-752 (2013).

17. Burke, C. W., Alexander, E. T., Timbie, K., Kilbanov, A. L. \& Price, R. J. Ultrasoundactivated agents comprised of 5FU-bearing nanoparticles bonded to microbubbles inhibit solid tumor growth and improve survival. Mol. Ther. 22, 321-328 (2014). 
18. Yan, F. et al. Paclitaxel-liposome-microbubble complexes as ultrasoundtriggered therapeutic drug delivery carriers. J. Control. Release 166, 246-255 (2013).

19. Escoffre, J. M., Novell, A., Serriere, S., Lecomte, T. \& Bouakaz, A. Irinotecan delivery by microbubble-assisted ultrasound: in vitro validation and a pilot preclinical study. Mol. Pharm. 10, 2667-2675 (2013).

20. Kooiman, K., Vos, H. J., Versluis, M. \& de Jong, N. Acoustic behavior of microbubbles and implications for drug delivery. Adv. Drug Deliv. Rev. 72 28-48 (2014)

21. Faez, T. et al. 20 years of ultrasound contrast agent modeling. IEEE Trans. Ultrason., Ferroelectr., Freq. Control 60, 7-20 (2013).

22. Liu, H. L., Fan, C. H., Ting, C. Y. \& Yeh, C. K. Combining microbubbles and ultrasound for drug delivery to brain tumors: current progress and overview. Theranostics 4, 432-444 (2014).

23. Klibanov, A. L., Shevchenko, T. I., Raju, B. I., Seip, R. \& Chin, C. T. Ultrasoundtriggered release of materials entrapped in microbubble-liposome constructs: a tool for targeted drug delivery. J. Control. Release 148, 13-17 (2010).

24. Hernot, S. \& Klibanov, A. L. Microbubbles in ultrasound-triggered drug and gene delivery. Adv. Drug Deliv. Rev. 60, 1153-1166 (2008).

25. Setyawati, M. I., Tay, C. Y., Bay, B. H. \& Leong, D. T. Gold nanoparticles induced endothelial leakiness depends on particle size and endothelial cell origin. ACS Nano 11, 5020-5030 (2017).

26. Tay, C. Y., Setyawati, M. I. \& Leong, D. T. Nanoparticle density: a critical biophysical regulator of endothelial permeability. ACS Nano 11, 2764-2772 (2017).

27. Setyawati, M. I., Mochalin, V. N. \& Leong, D. T. Tuning endothelial permeability with functionalized nanodiamonds. ACS Nano 10, 1170-1181 (2016).
28. Setyawati, M. I., Tay, C. Y., Docter, D., Stauber, R. H. \& Leong, D. T. Understanding and exploiting nanoparticles' intimacy with the blood vessel and blood. Chem. Soc. Rev. 44, 8174-8199 (2015).

29. Setyawati, M. I. et al. Titanium dioxide nanomaterials cause endothelial cell leakiness by disrupting the homophilic interaction of VE-cadherin. Nat Commun. 4, 1673 (2013)

30. Mayer, L. D. et al. Ratiometric dosing of anticancer drug combinations: controlling drug ratios after systemic administration regulates therapeutic activity in tumor-bearing mice. Mol. Cancer Ther. 5, 1854-1863 (2006).

31. Tardi, P. G. et al. Coencapsulation of irinotecan and floxuridine into low cholesterol-containing liposomes that coordinate drug release in vivo. Biochim. Biophys. Acta 1768, 678-687 (2007).

32. Harasym, T. O. et al. Increased preclinical efficacy of irinotecan and floxuridine coencapsulated inside liposomes is associated with tumor delivery of synergistic drug ratios. Oncol. Res. 16, 361-374 (2007).

33. Liang, $X$. et al. Self-assembly of an amphiphilic Janus camptothecin-floxuridine conjugate into liposome-like nanocapsules for more efficacious combination chemotherapy in cancer. Adv. Mater. 29, 1703135 (2017).

34. Gao, C. et al. Near-infrared cyanine-loaded liposome-like nanocapsules of camptothecin-floxuridine conjugate for enhanced chemophotothermal combination cancer therapy. ACS Appl. Mater. Interfaces 10, 3219-3228 (2018).

35. Liang, $X ., L i, X$., Yue, X. \& Dai, Z. Conjugation of porphyrin to nanohybrid cerasomes for photodynamic diagnosis and therapy of cancer. Angew. Chem. Int. Ed. Engl. 50, 11622-11627 (2011). 